\title{
Assessing perinatal anxiety: What to ask
}

Ashley B. Jones, MD, and Kaustubh G. Joshi, MD

E merging data demonstrate that untreated perinatal anxiety is associated with negative outcomes, including an increased risk for suicide. ${ }^{1}$ A 2017 systematic review and meta-analysis that included 102 studies with a total of 221,974 women from 34 countries found that the prevalence of self-reported anxiety symptoms and any anxiety disorder was $22.9 \%$ and $15.2 \%$, respectively, across the 3 trimesters. ${ }^{1}$ During pregnancy, anxiety disorders (eg, generalized anxiety disorder) and anxiety-related disorders (eg, obsessivecompulsive disorder [OCD] and posttraumatic stress disorder [PTSD]) can present as new illnesses or as a reoccurrence of an existing illness. Patients with pre-existing OCD may notice that the nature of their obsessions is changing. Women with preexisting PTSD may have their symptoms triggered by pregnancy or delivery or may develop PTSD as a result of a traumatic delivery. Anxiety is frequently comorbid with depression, and high anxiety during pregnancy is one of the strongest risk factors for depression. ${ }^{1,2}$

In light of this data, awareness and recognition of perinatal anxiety is critical. In this article, we describe how to accurately assess perinatal anxiety by avoiding assumptions and asking key questions during the clinical interview.

\section{Avoid these common assumptions}

Assessment begins with avoiding assumptions typically associated with maternal mental health. One common assumption is that pregnancy is a joyous occasion for all women. Pregnancy can be a stressful time that has its own unique difficulties, including the potential to develop or have a relapse of a mental illness. Another assumption is that the only concern is "postpartum depression." In actuality, a significant percentage of women will experience depression during their pregnancy (not just in the postpartum period), and many other psychiatric illnesses are common during the perinatal period, including anxiety disorders.

\section{Conduct a focused interview}

Risk factors associated with antenatal anxiety include ${ }^{2}$ :

- previous history of mental illness (particularly a history of anxiety and depression and a history of psychiatric treatment)

- lack of partner or social support

- history of abuse or domestic violence

- unplanned or unwanted pregnancy

- adverse events in life and high perceived stress

- present/past pregnancy complications - pregnancy loss.

Symptoms of anxiety. The presence of anxiety or worrying does not necessarily mean a mother has an anxiety disorder. Using the DSM- 5 as a guide, we should use the ques-

Every issue of CURRent Psychiatry has its 'Pearls'

Yours could be found here.

Read the 'Pearls' guidelines for manuscript submission at MDedge.com/ CurrentPsychiatry/page/pearls. Then, share with your peers a 'Pearl' of wisdom from your practice.
Dr. Jones is Associate Professor of Clinical Psychiatry, Adjunct Associate Professor of Clinical Obstetrics and Gynecology, and Director, General Psychiatry Residency, Prisma Health Midlands, Columbia, South Carolina. Dr. Joshi is Associate Professor of Clinical Psychiatry and Associate Director, Forensic Psychiatry Fellowship, Department of Neuropsychiatry and Behavioral Science, University of South Carolina School of Medicine, Columbia, South Carolina. He is one of CURRENT PsYCHATRY's Department Editors for Pearls.

\section{Disclosures}

The authors report no financial relationships with any companies whose products are mentioned in this article, or with manufacturers of competing products.

doi: 10.12788/cp.0127 
Merely asking

patients if

they have

had thoughts

of harming

their baby is

insufficient

\section{$f$}

Discuss this article at www.facebook.com/ MDedgePsychiatry tions outlined in the following sections to inquire about all of the symptoms related to a particular illness, the pervasiveness of these symptoms, and to what extent these symptoms impair a woman's ability to function and carry out her usual activities. ${ }^{3}$

Past psychiatric history. Ask your patient the following: Have you previously experienced anxiety and/or depressive symptoms? Were those symptoms limited only to times when you were pregnant or postpartum? Were your symptoms severe enough to disrupt your life (job, school, relationships, ability to complete daily tasks)? What treatments were effective for your symptoms? What treatments were ineffective? ${ }^{3}$

Social factors. Learn more about your patient's support systems by asking: Who do you consider to be part of your social support? How is your relationship with your social support? Are there challenges in your relationship with your friends, family, or partner? If yes, what are those challenges? Are there other children in the home, and do you have support for them? Is your home environment safe? Do you feel that you have what you need for the baby? What stressors are you currently experiencing? Do you attend support groups for expectant mothers? Are you engaged in perinatal care ${ }^{3}$

Given the high prevalence of interpersonal violence in women of reproductive age, all patients should be screened for this. The American College of Obstetricians and Gynecologists Committee on Health Care for Underserved Women recommends screening for interpersonal violence at the first visit during the perinatal period, during each trimester, and at the postpartum visit (at minimum). ${ }^{4}$ Potential screening questions include (but are not limited to): Have you and/or your children ever been threatened by or felt afraid of your partner? When you argue with your partner, do either of you get physical? Has your partner ever physically hurt you (eg, hit, choked)? Do you feel safe at home? Do you have a safe place to go with resources you and your children will need in case of an emergency ${ }^{4-6}$
Feelings toward pregnancy, past/current pregnancy complications, and pregnancy loss. Ask your patient: Was this pregnancy planned? How do you feel about your pregnancy? How do you see yourself as a mother? Do you currently have pregnancy complications and/or have had them in the past, and, if so, what are/were they? Have you lost a pregnancy? If so, what was that like? Do you have fears related to childbirth, and, if so, what are they? ${ }^{3}$

Intrusive thoughts about harming the baby. Intrusive thoughts are common in postpartum women with anxiety disorders, including OCD. ${ }^{7}$ Merely asking patients if they've had thoughts of harming their baby is incomplete and insufficient to assess for intrusive thoughts. This question does not distinguish between intrusive thoughts and homicidal ideation; this distinction is absolutely necessary given the difference in potential risk to the infant.

Intrusive thoughts are generally associated with a low risk of mothers acting on their thoughts. These thoughts are typically ego dystonic and, in the most severe form, can be distressing to an extent that they cause behavioral changes, such as avoiding bathing the infant, avoiding diaper changes, avoiding knives, or separating themselves from the infant. ${ }^{7}$ On the contrary, having homicidal ideation carries a higher risk for harm to the infant. Homicidal ideation may be seen in patients with co-occurring psychosis, poor reality testing, and delusions. ${ }^{5,7}$

Questions such as "Do you worry about harm coming to your baby?" "Do you worry about you causing harm to your baby?" and "Have you had an upsetting thought about harming your baby?" are more likely to reveal intrusive thoughts and prompt further exploration. Statements such as "Some people tell me that they have distressing thoughts about harm coming to their baby" can gently open the door to a having a dialogue about such thoughts. This dialogue is significantly important in making informed assessments as we develop comprehensive treatment plans. 


\section{References}

1. Dennis CL, Falah-Hassani K, Shiri R. Prevalence of antenatal and postnatal anxiety: systematic review and meta-analysis. B J Psychiatry. 2017;210(5):315-323.

2. Biaggi A, Conroy S, Pawlby S, et al. Identifying the women at risk of antenatal anxiety and depression: a systematic review. J Affect Disord. 2016;191:62-77.

3. Kirby N, Kilsby A, Walker R. Assessing low mood during pregnancy. BMJ. 2019;366:I4584. doi: 10.1136/bmj.I4584

4. American College of Obstetricians and Gynecologists Committee on Health Care for Underserved Women. Committee opinion: Intimate partner violence. Number
518. February 2012. Accessed March 23, 2020. https:/ / www. acog.org/clinical/clinical-guidance/committee-opinion/ articles/2012/02/intimate-partner-violence

5. Massachusetts Child Psychiatry Access Program for Moms Provider Toolkit. Accessed March 18, 2020. https://www.mcpapformoms.org/Docs/AdultProvider Toolkit12.09.2019.pdf

6. Ashur ML. Asking about domestic violence: SAFE questions. JAMA. 1993;269(18):2367.

7. Brandes $\mathrm{M}$, Soares $\mathrm{CN}$, Cohen LS. Postpartum onset obsessive-compulsive disorder: diagnosis and management. Arch Womens Ment Health. 2004;7(2):99-110. 